\title{
Cell biology needs super-resolution
}

\author{
Oliver von Bohlen und Halbach
}

The understanding of cellular functions requires detailed knowledge of all factors and their cellular distribution. Conventional microscopy has played (and still plays) a key role in cell biology. Microscopy combined with specific fluorescent labeling techniques has allowed to analyze the spatial distribution and/ or the dynamics of subcellular structures or protein of interest in detail. However, conventional microscopy is limited in resolution and therefore, structures smaller than $200 \mathrm{~nm}$ can hardly be visualized. The problem is that the maximum resolution of lens-based microscopy is constrained by the diffraction limit of light. During the transmission through an objective lens, diffraction limits the minimum size of a focal point and results in a three-dimensional point spread function (PSF) of the light. Thus, if two structures are too close together, their individual PSFs cannot be distinguished. Thus, the two points cannot be resolved. Roughly spoken, even under best conditions, the optical resolution of a light microscope is limited to approximately half of the wavelength of the light used. Therefore, only structures that were at least 200 to $350 \mathrm{~nm}$ apart (depending on the wave-length of the light) could be resolved by light microscopy [1]. Recently, several new technologies, termed "super-resolution microscopy" or "nanoscopy", have been developed that circumvent the diffraction limit and shift the optical resolution down to 20-100 $\mathrm{nm}$. Among these technologies are three different far-field

Oliver von Bohlen und Halbach

Affiliations: Institut für Anatomie und Zellbiologie, Universitätsmedizin Greifswald, Friedrich-Löffler-Straße-23c, 17487 Greifswald, Germany. Corresponding Author: Oliver von Bohlen und Halbach, Institute of Anatomy and Cell Biology, Universitätsmedizin, Friedrich Loeffler Strasse 23c, 17487 Greifswald, Germany $\mathrm{Ph}:$ ++49-(0)3834-86-5313; Fax: ++49-(0)3834-86-5302; Email: oliver.vonbohlen@uni-greifswald.de

Received: 02 July 2015

Published: 12 September 2015 methods, termed STED, SIM, and PALM/STORM, that have become popular.

Stimulated emission depletion microscopy (STED) relies on the depletion of the fluorophore's excited state to reduce the detection PSF. To obtain diffraction-unlimited images, STED assembles an image by scanning pixel by pixel with a laser and removes the out-of focus light using a pinhole in the optical path [2]. The resolution of a STED system in the $\mathrm{X}-\mathrm{Y}$ axis is about $20-100 \mathrm{~nm}[1,3]$.

Structured illumination microscopy (SIM) is based on conventional wide field fluorescence microscopy. An advantage of the SIM technique is that it does not require a special fluorescent probe for the resolution improvement [4]. SIM illuminates the entire field with a striped pattern of light. The excitation pattern mixes with the spatial pattern of the sample and produces an interference pattern (called "moiré fringe"). The excitation pattern is translated and rotated to generate a series of image with different moiré fringes. Since the illumination pattern is known, it can be mathematically removed from the moiré to gain access to the normally irresolvable higher resolution information in the sample [5]. The resolution of a SIM system in the $\mathrm{X}-\mathrm{Y}$ axis is about $100 \mathrm{~nm}[1,5]$. Interestingly, the SIM technique can also be used in the tree-dimensional space. 3D-SIM allows to obtain 3D-images with $\sim 100 \mathrm{~nm}$ lateral and $\sim 300 \mathrm{~nm}$ axial resolution $[4,5]$.

Photoactivation localization microscopy (PALM), and stochastic optical reconstruction microscopy (STORM) represent specific single-molecule localization techniques. These techniques rely on the localization of single fluorophores based on their intensity data. The main principle of these techniques is based on the possibility to turn a subgroup of sparsely-distributed fluorescent molecules in subsequent imaging cycles on and off with the result of obtaining few sparse emitting molecules per cycle and captured image [2]. Series of a few hundred or thousand images can be processed into high-resolution images reaching a resolution in the range of about $30 \mathrm{~nm}$ [1].

Currently, the development of super-resolution microscopy just has started and will continue to evolve. Super-resolution microscopy has impressively improved 


\section{EDORIUM Journals}

Edorium J Cell Biol 2015;2:1-3.

von Bohlen und Halbach

the resolution in the $\mathrm{X}-\mathrm{Y}$ axis. However, it should be kept in mind that biological structures are three-dimensional. Therefore, there is a need to extend super-resolution microscopy to $3 \mathrm{D}$. In addition, image resolution often comes at the price of acquisition-speed and living cells may transport molecules faster than the superresolution systems may capture images. Super-resolution microscopy in general will evolve as well as specialized system will be developed that will focus on higher resolution, high sampling speed or specific acquisition within thick tissues.

Super-resolution technologies offer the opportunity to localize proteins within the cell very precisely without the need for electron microscopy. Super-resolution microscopy will also allow to study small subcellular structures that have a length or width near and/or below the diffraction limit of the light, as e.g., the dendritic spines of a neuron. Thus, super-resolution microscopy has been successfully used to determine the nanoscopic spine localization of a mGluR 5 accessory protein, called Norbin [6]. Moreover, by using PALM in combination with quantum dot tracking, morphological dynamic changes in spine morphology have been analyzed [7] .

Although these technologies are currently not widely used for biological research, current super-resolution approaches allow fascinating new insights in cellular structures. The future developments in the field of superresolution microscopy will have the power to revolutionize biological research.

Keywords: Cell biology, Nanoscopy, Photoactivation localization microscopy, Structured illumination microscopy (SIM), Stimulated emission depletion microscopy (STED), Super-resolution microscopy

How to cite this article

von Bohlen und Halbach O. Cell biology needs superresolution. Edorium J Cell Biol 2015;2:1-3.

Article ID: 100003Co6OH2O15

$* * * * * * * * *$

doi:10.5348/Co6-2015-3-ED-1

\section{Guarantor}

The corresponding author is the guarantor of submission.

\section{Conflict of Interest}

Authors declare no conflict of interest.

\section{Copyright}

(C) 2015 Oliver von Bohlen und Halbach. This article is distributed under the terms of Creative Commons Attribution License which permits unrestricted use, distribution and reproduction in any medium provided the original author(s) and original publisher are properly credited. Please see the copyright policy on the journal website for more information.

\section{REFERENCES}

1. Schermelleh L, Heintzmann R, Leonhardt H. A guide to super-resolution fluorescence microscopy. J Cell Biol 2010 Jul 26;190(2):165-75.

2. Fornasiero EF, Opazo F. Super-resolution imaging for cell biologists: concepts, applications, current challenges and developments. Bioessays 2015 Apr;37(4):436-51.

3. Hell SW. Far-field optical nanoscopy. Science 2007 May 25;316(5828):1153-8.

4. Yamanaka M, Smith NI, Fujita K. Introduction to super-resolution microscopy. Microscopy (Oxf) 2014 Jun;63(3):177-92.

5. Galbraith CG, Galbraith JA. Super-resolution microscopy at a glance. J Cell Sci 2011 May 15;124(Pt 10):1607-11.

6. Westin L, Reuss M, Lindskog M, Aperia A, Brismar H. Nanoscopic spine localization of Norbin, an mGluR5 accessory protein. BMC Neurosci 2014 Mar 26;15:45.

7. Frost NA, Lu HE, Blanpied TA. Optimization of cell morphology measurement via single-molecule tracking PALM. PLoS One 2012;7(5):e36751.

\section{Author Contributions}

Oliver von Bohlen und Halbach - Substantial contribution to conception and design of the experiment, Data acquisition, Analysis and interpretation, Drafting the article, Final approval of the version to be published 


\section{EDORIUM Journals}

Edorium J Cell Biol 2015;2:1-3.

Access full text article on other devices

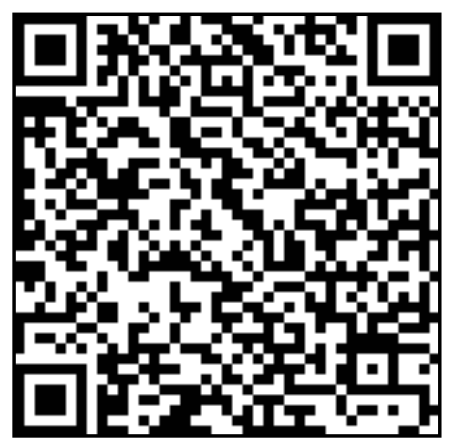

Access PDF of article on other devices

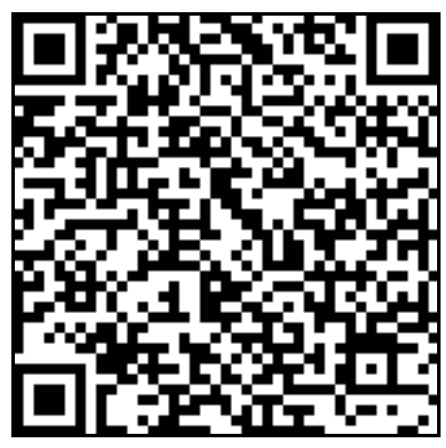

